\title{
Changes in the gut microbiota of cloned and non-cloned control pigs during development of obesity: gut microbiota during development of obesity in cloned pigs
}

Rebecca Pedersen ${ }^{1}$, Anders Daniel Andersen ${ }^{1}$, Lars Mølbak,3, Jan Stagsted ${ }^{2}$ and Mette Boye ${ }^{1 *}$

\begin{abstract}
Background: Obesity induced by a high-caloric diet has previously been associated with changes in the gut microbiota in mice and in humans. In this study, pigs were cloned to minimize genetic and biological variation among the animals with the aim of developing a controlled metabolomic model suitable for a diet-intervention study. Cloning of pigs may be an attractive way to reduce genetic influences when investigating the effect of diet and obesity on different physiological sites. The aim of this study was to assess and compare the changes in the composition of the gut microbiota of cloned vs. non-cloned pigs during development of obesity by a high-fat/ high-caloric diet. Furthermore, we investigated the association between diet-induced obesity and the relative abundance of the phyla Firmicutes and Bacteroidetes in the fecal-microbiota. The fecal microbiota from obese cloned $(n=5)$ and non-cloned control pigs $(n=6)$ was investigated biweekly over a period of 136 days, by terminal restriction fragment length polymorphism (T-RFLP) and quantitative real time PCR (qPCR).
\end{abstract}

Results: A positive correlation was observed between body-weight at endpoint and percent body-fat in cloned $(r=0.9, P<0.0001)$ and in non-cloned control pigs $(r=0.9, P<0.0001)$. Shannon Weaver and principal component analysis (PCA) of the terminal restriction fragments (T-RFs) revealed no differences in the bacterial composition or variability of the fecal microbiota between the cloned pigs or between cloned and non-cloned control pigs. Body-weight correlated positively with the relative abundance of Firmicutes in both cloned $(r=0.37 ; P<0.02)$ and non cloned-control pigs $(r=0.45 ; P<0.006)$, and negatively with the abundance of Bacteroidetes in cloned pigs $(r=-0.33$, $P<0.04)$, but not in the non-cloned control pigs.

Conclusion: The cloned pigs did not have reduced inter-individual variation as compared to non-cloned pigs in regard to their gut microbiota in neither the obese nor the lean state. Diet-induced obesity was associated with an increase in the relative abundance of Firmicutes over time. Our results suggest that cloned pigs are not a more suitable animal model for gut microbiota-obesity related studies than non-cloned pigs. This study is the first to evaluate if cloned pigs provide a better animal model than conventional pigs in diet-intervention, obesity and gut microbiota research.

Keywords: Gut microbiota, Cloned pigs, Diet-induced obesity, Bacterial diversity, Bacteroidetes, Firmicutes

\footnotetext{
*Correspondence: mboy@vet.dtu.dk

${ }^{1}$ National Veterinary Institute, Technical University of Denmark, Bülowsvej 27, Frederiksberg C 1870, Denmark

Full list of author information is available at the end of the article
} 


\section{Background}

Obesity and its associated morbidities have become an increasing problem in many countries around the world. While traditionally regarded as primarily a question of a sedentary lifestyle in which energy intake exceeds energy expenditure, new studies also point to the composition of the intestinal microbiota as a potentially contributing factor. In studies of diet-induced obesity and its association with the gut microbiota, it may be preferable to eliminate the influence of host genotype on the composition of the gut microbiota by choosing genetically identical animals. Some early investigations comparing the composition of the microbiota in human mono-zygotic twins (MZ) with di-zygotic twins (DZ) reported that the host genome was influencing the microbial composition in the gut $[1,2]$. A similar study based on 16S rRNA gene analysis indicated that bacterial community in human MZ twins was slightly more similar than in unrelated individuals [3] suggesting that genetically identical individuals harbor a similar gut microbiota. In a more recent study on the relationship between gut microbiota, diet and genetic influences in mice, the authors stated that the changes in gut microbiota were unrelated to genetically induced obesity and were merely due to high-fat (HF) diet [4]. Therefore, the influence of the host genome on the gut microbiota currently remains controversial.

When choosing an animal model for studying human diseases, it is important to choose animals that physiologically resemble humans. Pigs are good models for humans, primarily due to close resemblance of their anatomy and physiology of the digestive system and because pigs are omnivorous like humans $[5,6]$. Consequently, pigs are widely used in studies of human lifestyle-related diseases such as diabetes, cardiovascular disease and metabolic syndrome $[7,8]$. Using cloned pigs in obesity-related studies could provide a more homogenous experimental model, hence the cloning in this study was performed to minimize genetic influences and thereby reduce interindividual variation [9].

One of the main focuses of obesity-related gut microbial studies have been to identify groups of bacteria that are correlated with the obese state, and initially the relative abundance of Bacteroidetes and Firmicutes in the gut microbiota was linked to obesity. In pigs, as in humans [10] and other mammals [11], the two main phyla of bacteria in the gut microbiota are Bacteroidetes and Firmicutes [12,13]. Previous studies have reported a greater proportion of Firmicutes in obese mice [14] when compared with their leaner counterparts and a reduced ratio of Firmicutes to Bacteroidetes in a small group of obese humans on a weight loss regimen [15]. A similar result in a study of lean and obese pigs revealed a negative correlation between percentage of Bacteroidetes and body-weight [16]. Furthermore, a fluorescence in situ hybridization (FISH)-based study on obese adolescents during weight loss regimens showed a decrease in the phylum Firmicutes [17]. However several studies suggest a decrease in ratio of Firmicutes to Bacteroidetes in obese and overweight subjects [18] and suggest diet to be a contributing factor in shaping the gut microbial community and not the bacterial proportions $[19,20]$. Other observations in humans, suggest obesity to be associated with a lower bacterial diversity [3], while other studies showed no difference in the abundance of bacteria in the gut microbiota between lean and obese individuals that were on weight maintaining diet [21]. Hence this putative relationship between obesity, diet and specific phyla of bacteria in the gut microbiota is still controversial and there are few studies on the association between the gut microbiota and obesity during the development of obesity. Therefore, the focus of this paper was to investigate the gut microbiota in cloned pigs compared with non-cloned control pigs and to further elucidate if diet-induced obesity over time is associated with changes in the gut microbiota. We hypothesized that the composition of the gut microbiota would be more similar among the cloned pigs compared to non-cloned controls. The second hypothesis was that weight-gain would be related to an increase in the ratio of Firmicutes to Bacteroidetes as well as a decrease in the diversity of the gut microbiota. We therefore investigated the changes in the gut microbiota of cloned and control pigs beginning with lean pigs during a period of 136 days on a high-fat/high-caloric (HF/high-caloric) diet.

\section{Methods}

\section{Animals}

The animals for this experiment were pigs of similar genotype of Danish Landrace and Yorkshire. Six female siblings from a normal litter (the control group) (75\% Landrace $\times 25 \%$ Yorkshire) were obtained after standard artificial insemination followed by caesarian section. The cloning experiments were performed using donor cells obtained from a $65 \%$ Landrace $\mathrm{x} 35 \%$ Yorkshire sow as described previously [9]. The cloned embryos were then transferred surgically to surrogate sows (recipients) five to six days after cloning [9]. Two surrogate sows gave birth to five live female clones by caesarean section. Pigs were reared in the experimental stables at University of Aarhus (Tjele, Denmark). All the experimental animal studies were approved by the Danish Animal Experimental Committee.

\section{Experimental set up and sample collection}

The pigs in the experiment were weaned at 28 days of age and subsequently fed a standard pig-diet with an energy distribution of $18.5 \%$ protein, $7.9 \%$ fat, $72.4 \%$ carbohydrate and $1.2 \%$ fiber, for approximately 61 days. During this post weaning period animals from the same 
litter were housed together in the same stable. At 96 days (cloned pigs) and 89 days (non-cloned controls) of age (baseline), the pigs were transferred to facilities for individual housing and fed a wheat-based HF/highcaloric diet consisting of $19.5 \%$ protein, $27 \%$ fat, $53 \%$ carbohydrates and $0.5 \%$ fiber [22] with ad libitum access to the feed in order to induce obesity. The feed was weighed before and after feeding and the pigs were maintained on this diet for a period of 136 days until they were euthanized. The cloned and non-cloned control pigs were weighed biweekly starting a day prior to switch to HF/high-caloric feed and the body-fat composition of the animals was measured by computed tomography $(\mathrm{CT})$ scan at the end of the experiment. During this period, fresh feces collected biweekly were snapfrozen in liquid nitrogen and stored at $-20^{\circ} \mathrm{C}$ until later analyses.

\section{Terminal restriction fragment length polymorphism (T-RFLP)}

The fecal microbiota from all the pigs were analyzed by terminal restriction fragment length polymorphism (T-RFLP) fingerprint profiles as described previously [23]. In brief, DNA was extracted from $200 \mathrm{mg}$ feces by using the QIAamp DNA Stool Mini Kit (Qiagen, Hilden, Germany) according to manufacturer's instructions, with an additional step of bead beating in order to disrupt the cell wall of Gram-positive bacteria. The concentrations of DNA were measured in each sample by a spectrophotometer and adjusted to $5 \mathrm{ng} \mathrm{\mu l}^{-1}$ (NanoDrop Technologies,Wilmington, DE, USA). Amplification of $16 \mathrm{~S}$ rRNA gene DNA were performed in duplicates by using $16 \mathrm{~S}$ rRNA gene DNA bacterial specific primers, Eub-8fm (5'- AGAGTTTGATCMTGGCTCAG- 3') labeled with 5' FAM and Eub-926r (5-'CCGTCAATTCCTTTRAGT TT- 3') (DNA Technology, Aarhus, Denmark) [23]. Each PCR mix contained $5 \mu$ l of 10x Fermentas Taq-buffer, $4 \mu \mathrm{l}$ $\mathrm{MgCl}_{2}, 2.0 \mu \mathrm{l}$ deoxyribonucleotide triphosphate (dNTP), $0.5 \mu \mathrm{l}$ Fermentas Taq-polymerase, $0.5 \mu \mathrm{l}$ of each primer and $35.5 \mu \mathrm{l}$ nuclease-free water and $5 \mathrm{ng} \mathrm{ul}^{-1}$ DNA (final concentration of $0.2 \mathrm{ng}$ ). The cycling conditions were: initial denaturation at $94^{\circ} \mathrm{C}$ for 6 minutes (min) followed by 32 cycles of denaturing at $94^{\circ} \mathrm{C}$ for 45 seconds (s), annealing at $56^{\circ} \mathrm{C}$ for $45 \mathrm{~s}$, an extension step at $72^{\circ} \mathrm{C}$ for $2 \mathrm{~min}$, and a final extension at $72^{\circ} \mathrm{C}$ for $10 \mathrm{~min}$. The PCR products were subsequently verified by gel electrophoresis and purified by High Pure PCR Purification Kit (Roche Applied Sciences, Mannheim, Germany). The purified PCR product (200 ng) was digested with $2.0 \mu \mathrm{l}$ of the restriction enzyme HhaI (Promega Corporation, Madison, USA) at $37^{\circ} \mathrm{C}$ for $3 \mathrm{~h}$. Two $\mu \mathrm{l}$ of the digested PCR products, $10 \mu \mathrm{l}$ formamide and $0.50 \mu \mathrm{l}$ Megabase ET900-R Size Standard (GE Health Care, Buckinghamshire, UK) were mixed and run in duplicates on a capillary elec- trophoresis genetic analyzer (Genetic Analyzer 3130/ 3130xl, Applied Biosystems, Carlsberg, CA). The terminal restriction fragments (T-RFs), representing bacterial fragments in base pair (bp), were obtained and the analysis of T-RF profiles and alignment of T-RFs against an internal standard was performed using the BioNumerics software version 4.5 (Applied Maths, Kortrijk, Belgium).

T-RF fragments (range of 60-800 bp) with a difference less than two base pairs were considered identical. Only bands present in both duplicates were accepted as bacterial fragments from which the duplicate with the best intensity was chosen for microbial profiling. The obtained intensities of all T-RFs were imported into Microsoft Excel, and all intensities below 50 were removed. In each sample, the relative intensity of any given T-RF was calculated by dividing the intensity of the T-RF with the total intensity of all T-RFs in the sample. The most predominant T-RFs with a mean relative intensity above one percent were selected for all further analyses and procedures (except calculation of the diversity and similarity) and their identity was predicted in silico, performed in the MiCA on-line software [24] and Ribosomal Database Project Classifier (322.864 Good Quality, >1200) [25].

\section{T-RFLP statistical analysis}

All T-RFs between 60 and 800 bp were imported into the statistical software programs Stata 11.0 (StataCorp, College Station, TX), Unscrambler version 9.8 (CAMO, Oslo, Norway) and Microsoft Excel sheets were used for further analyses. Principal component analysis (PCA) was used to explore group differences in the overall microbial communities both for comparisons between cloned pigs and non-cloned controls at the different sampling points and to investigate if samples from pigs with the largest weight-gain during the study period clustered together, irrespective of their genetic background. The latter was also investigated by relating the whole microbial community to the weight-gain at the different sampling points, involving all predominant T-RFs simultaneously in the models. For this purpose partial least square regression (PLS-R) was used, which is a supervised model, meaning in this case that the variation in the weight (gain) data is used to actively decompose the variation in the bacterial data. In both analyses, the T-RFs were standardized (centered and 1/SD) prior to the modeling phase to ensure that all of them would equally influence the models, and possible outliers were inspected visually and with Hotelling $T^{2}$.

The diversity index was calculated as described previously [26]. In brief, the Shannon-Weaver index of diversity $\left(H^{\prime}\right)$ based on all of the initial T-RFs was used to determine the diversity of the bacterial fragments. Group comparisons of the diversity index in cloned versus non- 
cloned controls were calculated at each of the sampling points. As the Shannon-Weaver index was not normally distributed, Mann Whitney $U$ test and Spearman correlation were applied. The $H^{\prime}$ values are represented in figures as mean and error bars representing standard deviations (SD). Dice similarity between groups based on all the T-RFs were calculated in BioNumerics (Applied Maths, Kortrijk, Belgium) and the results are presented as mean values. T-RFs in the figures are presented as mean and standard error of the mean (SEM). A significant difference was considered when P-value was less than 0.05 $(P<0.05)$.

\section{Fecal samples and bacterial strains for $\mathrm{QPCR}$}

The extracted DNA from the fecal samples used for the T-RFLP analyses were also analyzed by qPCR, but only samples taken monthly were chosen for qPCR analysis. However additional sampling points two weeks before the endpoint samples were also analyzed by qPCR. Three bacterial strains (Clostridium perfringens (NCTC 8449), Odoribacter splanchnicus (isolate DJF_B089) and Escherichia coli (ATCC 25922), representing the Firmicutes and Bacteroidetes phyla and general bacteria, respectively, and six randomly chosen extracted DNA samples (divided equally into clones and controls) were used to optimize the PCR conditions.

\section{qPCR primers and conditions}

The 16S rRNA gene DNA primers for Bacteroidetes and Firmicutes used in this study were designed by Baccetti De Gregoris et al. [27] and conditions were optimized for the thermocycler used (Rotor-Gene Q Real Time PCR cycler (Qiagene)). The universal primer used in this study had an amplicon length of 147 bp (S-D-Bact-09 07-a-S-20 5'-AAACTCAAAGGAATTGACGG-3'; S-DBact-1054-a-A-20 5-' ACGAGCTGACGACAGCCATG-3') [12]. The specific primer sets for Bacteroidetes (798cfbF 5' CRAACAGGATTAGATACCCT'3 and cfb967R 5' GGTA AGGTTCCTCGCGTAT '3) and Firmicutes (928F-Firm 5' TGAAACTYAAAGGAATTGACG '3; 1040firmR, 5’ ACC ATGCACCACCTGTC '3) had an amplicon length of 240 bp and 200 bp, respectively [27]. All qPCR reactions contained $12.5 \mu$ l of SYBR $^{\circledR}$ Green JumpStart ${ }^{\mathrm{TM}}$ Taq ReadyMix ${ }^{\mathrm{TM}}$ without $\mathrm{MgCl}_{2}$ (Sigma-Aldrich, Copenhagen, Denmark), $0.3 \mu \mathrm{mol} \mathrm{l}^{-1}$ of each primer and $5 \mu \mathrm{l}$ of template DNA adjusted to $5 \mathrm{ng} \mathrm{\mu l}^{-1}$. $\mathrm{MgCl}_{2}$ optimization was performed and a final concentration of $2.5 \mathrm{mM} \mathrm{MgCl} 2$ was chosen. The annealing temperature was optimized by using $16 \mathrm{~S}$ rRNA gene DNA extracted from fecal samples and DNA extracted from different bacteria. Subsequently, all the primers and other PCR conditions were verified by conventional PCR and gel electrophoresis. A non template control (NTC) was included in each run. qPCR was performed with an initial denaturing step of $10 \mathrm{~min}$ at $95^{\circ} \mathrm{C}$, $95^{\circ} \mathrm{C}$ for $30 \mathrm{~s}, 35$ cycles of $56^{\circ} \mathrm{C}$ for $20 \mathrm{~s}$ and an elongation step of $72^{\circ} \mathrm{C}$ for $20 \mathrm{~s}$. A melting curve analysis was performed after each run to detect any primer-dimers in each sample. The threshold cycle $\left(C_{T}\right)$ and calculated concentrations (copies $\mu^{-1}$ ) were determined automatically by the Rotor Gene software (Rotor-Gene Q 2.0.2 (Qiagene)).

\section{Analysis of data from qPCR}

qPCR was performed to quantify relative abundance of the phyla Bacteroidetes and Firmicutes, respectively, present in each sample. The measured bacterial copy numbers of the 16S rRNA gene from bacteria belonging to the phylum Bacteroidetes and the phylum Firmicutes were calculated against $16 \mathrm{~S}$ rRNA genes obtained from all bacteria and the relative abundance of the two phyla in each sample was subsequently calculated and statistically evaluated by Mann Whitney $U$ test. Further correlation analyses were performed using Spearman correlation coefficient and $P<0.05$ was considered statistically significant. A standard curve was constructed for specific and universal primer sets and assays using tenfold serial dilutions of the extracted DNA from $C$. perfringens, O. splanchnicus and $E$. coli all DNA samples in the range $2.5 \times 10^{2} \mathrm{ng} \mu \mathrm{L}^{-1}$ to $2.5 \times 10^{-6} \mathrm{ng} \mu \mathrm{L}^{-1}$. Furthermore, serial dilutions corresponding to the previously described dilutions of genomic DNA from two random samples were used to construct standard curves to further verify if PCR inhibitors were present in extracted DNA from fecal samples.

\section{Results}

\section{Weight of the animals}

At baseline, just before the animals were transferred to the ad libitum high-fat (HF)/high-caloric diet, the cloned (96 days old) and non-cloned control (89 days old) pigs weighed $38 \pm 4.1 \mathrm{~kg}$ (Mean \pm SEM) and $37.9 \pm 2.3 \mathrm{~kg}$, respectively. Daily weight-gain in cloned pigs $(\mathrm{n}=5)$ was $0.78 \pm 0.04 \mathrm{~kg}$ and in control pigs $(\mathrm{n}=6) 1.05 \pm 0.03 \mathrm{~kg}$, corresponding to a lower daily feed intake by cloned pigs than the controls. The clones weighed $143.6 \pm 8.8 \mathrm{~kg}$ at the time they were euthanized (end point), compared to control pigs, which weighed significantly more $(179.5 \pm$ $4.0 \mathrm{~kg}$ ) at the end of the study (difference of $35.9 \mathrm{~kg}$, $P=0.004)$. CT scanning of body fat showed that obese non-cloned control pigs had a higher average percentage of body-fat $(41.1 \pm 1.3 \%)$ than obese cloned pigs $(28.4 \pm$ $2.3 \%, P=0.004)$. There was a positive correlation between body-fat percentage and body weight at the end of the diet-intervention study in non-cloned control pigs as well as in cloned pigs $(\mathrm{r}=0.85, P=0.0001)$ (Figure 1).

\section{The compositional diversity of the gut microbiota}

The PCA analysis of the overall composition of the gut microbiota in all animals did not reveal separate clustering of the T-RF profiles between the cloned pigs and 


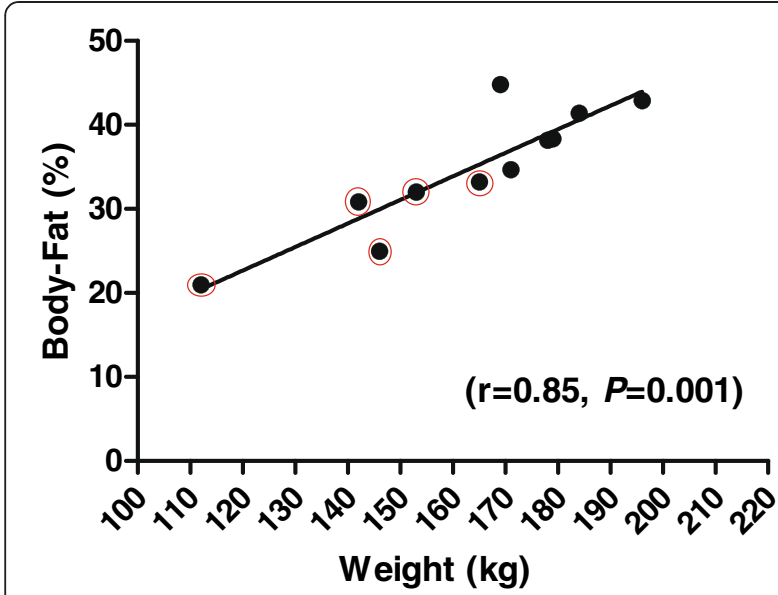

Figure 1 Correlation between percent body-fat and bodyweight $(\mathbf{k g})$. The correlation between percent body-fat and bodyweight $(\mathrm{kg})$ in all the pigs were calculated by Spearman correlation $(r=0.85, P=0.0001)$. The red circles indicate the cloned pigs and the non-cloned pigs are indicated by plain black dots.

the non-cloned controls. To test if the gut microbiota between cloned pigs was more similar than between non-cloned control pigs, a dice similarity score was calculated showing that the microbiota in cloned pigs was neither more uniform within the group nor more diverse compared to non-cloned control pigs (Figure 2A). Furthermore, there was no difference in Shannon-Weaver index between cloned and non-cloned control pigs at the start of diet-intervention (baseline), with ShannonWeaver index $\left(H^{\prime}\right), H^{\prime}=2.6(2.3-2.8)$ and $H^{\prime}=1.7$ (1.5-2.8), respectively. Within the control group, a slight increase $(P=0.01)$ in the diversity of the gut microbiota was observed from baseline to end of diet-intervention (end point) $\left(H^{\prime}=3,2.3-3.4\right)$, while no difference was observed in the cloned pig group ( $\left.H^{\prime}=3.3,2.3-3.4\right)$ (Figure 2B). Furthermore, there was no correlation between diversity of microbial community as found by Shannon-Weaver index and weight-gain (Figure 2B).

The bacterial load (including all initial T-RFs between 60 and $800 \mathrm{bp})$ in the fecal microbiota of cloned pigs and non-cloned control pigs was similar throughout the intervention period, both at baseline and at endpoint $(P=0.08$ and $P=0.3$, respectively). In general, the T-RF profiles were similar in the cloned pigs and non-cloned pigs (Figure $3 \mathrm{~A}$ and $\mathrm{B}$ ). Both cloned pigs and noncloned control pigs had 11 T-RFs with a relative abundance larger than one-percent in common at baseline and $17 \mathrm{~T}$-RFs at endpoint (Figure $3 \mathrm{~A}$ and $\mathrm{B}$ ). There were several differences in T-RFs between the cloned pigs and non-cloned control pigs, however these were not significant $(P=0.08)$.

In the non-cloned control group, one individual T-RF with a length of $102 \mathrm{bp}$ was found higher at baseline compared to endpoint $(P=0.04)$ (Figure $3 \mathrm{~B})$ and within
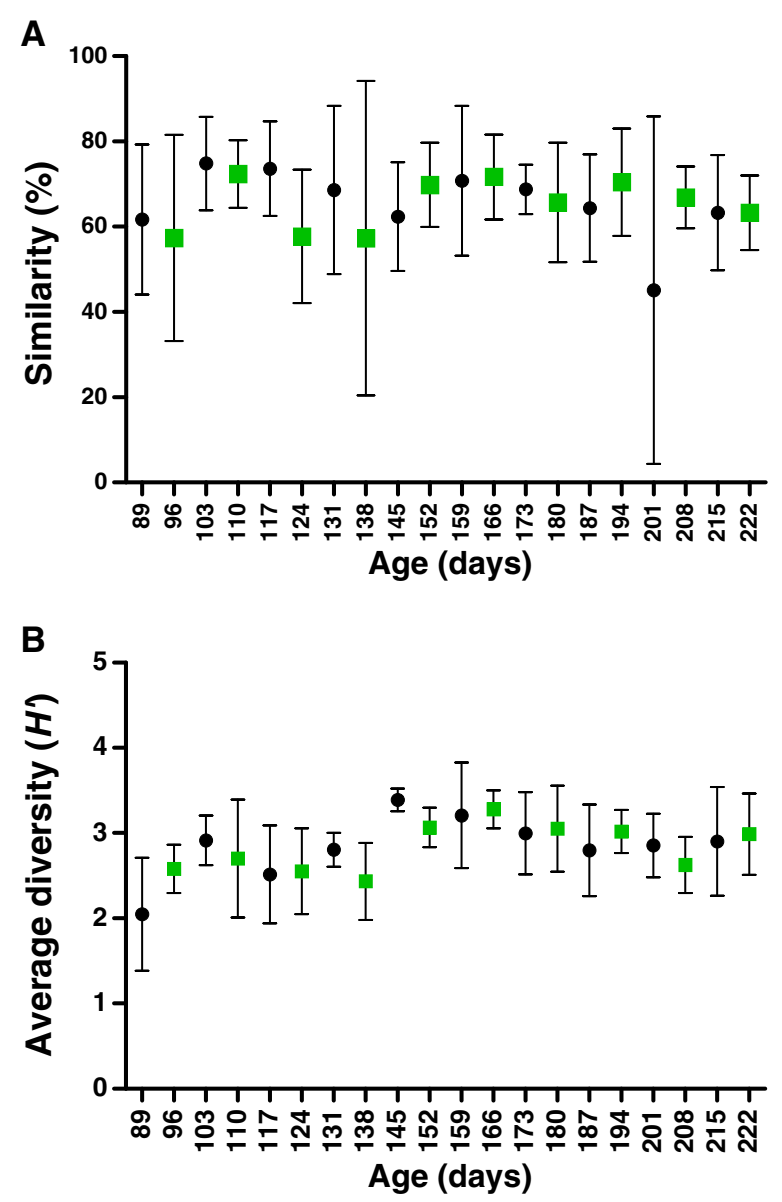

Figure 2 Similarity (A) and diversity (B) of gut microbiota. The similarity and diversity was calculated based on T-RFs (bp) at different age interval in non-cloned control pigs $(\bullet)$ and cloned pigs (green square) by Dice similarity index and Shannon-Weaver index. Results are presented in mean and the error bars represent standard deviations (SD).

the cloned pig group one T-RF (93 bp) was higher at endpoint than at baseline $(P=0.01)$ (Figure $3 \mathrm{~A})$. At baseline in the non-cloned control group, the relative abundance of T-RF $93 \mathrm{bp}$ was less than one percent and a significant increase in T-RF 93 bp from baseline to endpoint $(P=0.005)$ was observed. The in silico analysis of the obtained T-RFs indicated that the T-RF $93 \mathrm{bp}$ and T-RF 102 bp may be bacterial fragment belonging to the phylum Bacteroidetes (See Additional file 1).

At baseline a total of $47 \mathrm{~T}$-RFs were present in the cloned pigs fecal microbiota while at endpoint there were 85 T-RFs present, indicating a more rich community at endpoint. At baseline 27 T-RFs with intensities of more than $1 \%$ are represented in Figure 3A. Together these 27 T-RFs represent $92 \%$ of the all the T-RFs present at baseline.

In non-cloned control pigs, a total of 42 T-RFs were present at baseline and $85 \mathrm{~T}$-RFs were present at endpoint, 

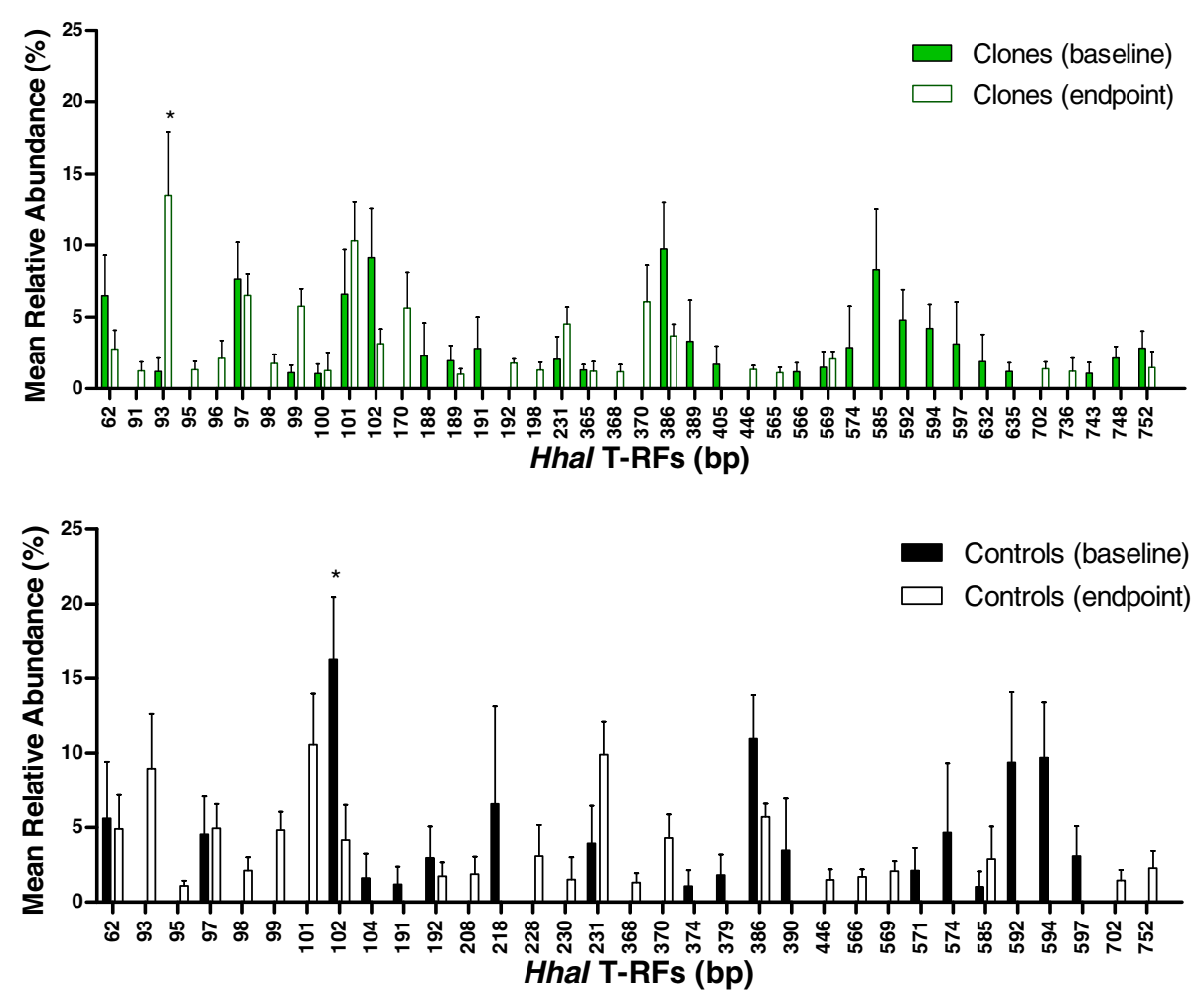

Figure 3 The abundance of bacteria at baseline and endpoint. Mean relative abundance of the most predominant T-RFs (>1\%, bp) in the fecal samples of cloned pigs at baseline (green square) and endpoint ( $\square$ ) and in non-cloned control pigs at baseline ( $(\mathbf{})$ ) and endpoint ( $\square$ ). The error bars represent standard error of the mean (SEM).

again indicating an increase in T-RFs from baseline to endpoint. At baseline, only 18 T-RFs had intensities larger than $1 \%$. These 18 T-RFs however, constituted $96 \%$ of all the T-RFs at baseline. At endpoint, there were $82 \mathrm{~T}-\mathrm{RFs}$ present in fecal microbiota of non-cloned pigs of which only 22 T-RFs had intensities of more than 1\% (Figure 3B). The possible identification of these T-RFs as found by in silico analysis, can be found in the supplementary material (See Additional file 1).

\section{Relative abundance of Firmicutes and Bacteroidetes in the gut microbiota by qPCR}

There was no difference in the relative abundance of Bacteroidetes between cloned and non-cloned control pig at baseline $(P=0.1)$ or at endpoint $(P=0.9)$ and the same was observed for Firmicutes (baseline, $P=0.8$; endpoint, $P=0.7$ ).

In cloned pigs, a negative correlation was observed between weight-gain and relative abundance of Bacteroidetes $(\mathrm{r}=-0.33, P<0.04)$ (Figure 4A). A continuous and significant decrease $(P<0.008)$ was observed in phylum Bacteroidetes from baseline and throughout the weightgain period (Figure 4A) which then began to rise again by the time the pigs had an average weight of $118.9 \pm 3.2 \mathrm{~kg}$ until the animals were euthanized at endpoint.
In the non-cloned control pigs, there was a decrease in the relative abundance of Bacteroidetes from baseline (weight: $37.9 \pm 2.3 \mathrm{~kg}$ ) until the pigs weighed $95.5 \pm 3.9 \mathrm{~kg}$, from which point the relative abundance of Bacteroidetes began to increase again until endpoint (Figure 4B). Subsequently, there was no significant difference in the relative abundance of Bacteroidetes at baseline and endpoint in the non-cloned pigs (Figure 4B).

In cloned pigs, an increase in relative abundance of Firmicutes was observed from baseline to endpoint $(P<0.009)$ (Figure $4 C)$ and the same was observed in noncloned control pigs from baseline to endpoint $(P<0.0001)$ (Figure 4D). This positive correlation between the weightgain and the relative abundance of Firmicutes during the study period was observed both in cloned pigs $(\mathrm{r}=0.37$, $P<0.02)$ and non-cloned control pigs $(\mathrm{r}=0.45, P<0.006)$ (Figure $4 \mathrm{C}$ and D, respectively). Additional figure shows the changes in the relative abundance of Firmicutes and Bacteroidetes during weight-gain (See Additional file 2).

\section{Discussion}

In order to establish a better understanding of the underlying causes of obesity and the effect of obesity on different body sites, the cloned pigs and non-cloned control pigs employed for our study were also investigated 


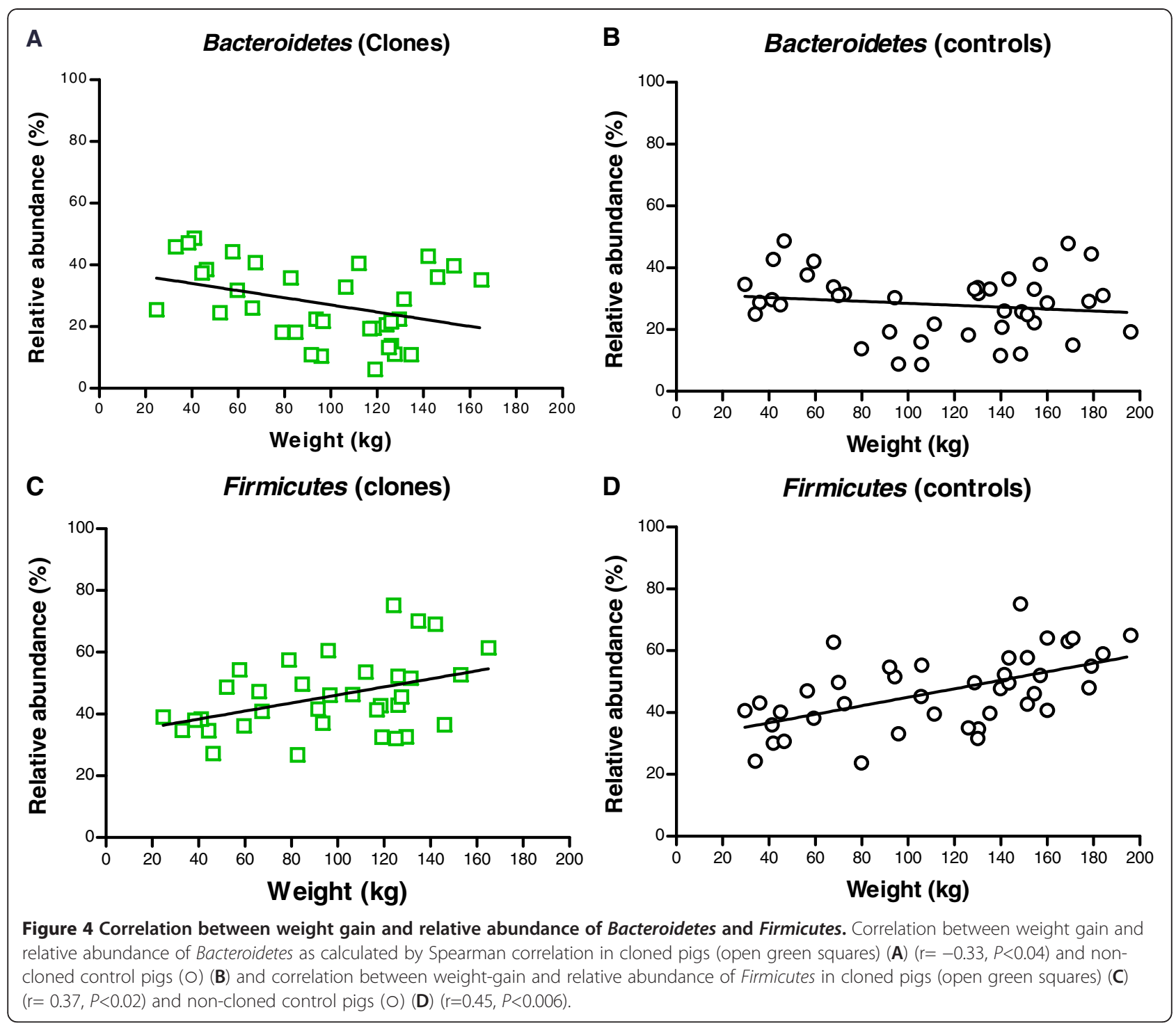

in regard to their immunological [28], metabolomics [22] and phenotypic characters [9]. In this study, we investigated the gut microbiota of both cloned and noncloned control pigs by T-RFLP and found that the gut microbiota within a group of five obese clones was neither more similar nor more diverse than the microbiota within a group of six obese non-cloned control pigs of the same sex and genetic background. The metabolomic phenotyping [9] of these obese cloned and non-cloned control pigs showed that the phenotype of the cloned pigs was different from the phenotype of non-cloned control pigs [9] and that the inter-individual variation amongst these cloned pigs was not less than the interindividual variation of the non-cloned control pigs that were siblings [22]. Hence, based on these and the findings presented in the current paper it would appear that the cloned pigs do not have identical phenotypes or less inter-individual variation than conventional non- cloned pigs. One explanation for these results could be that in cloning by somatic cell nuclear transfer the animals inherit maternal mitochondrial DNA and even though they have the same somatic DNA, the cloned pigs possess altering phenotypes due to the maternal mitochondrial DNA effect [9]. This raises the question of whether cloned animals are more suitable animal models than conventional non-cloned animals.

The heritable component of an individual and its effect on the microbial community have been investigated before in several human studies; in particular MZ twins have been investigated to minimize the genetic influence in order to get a better understanding of the role of obesity on gut microbiota [3]. When designing an experimental model for gut microbiota related studies, it is important to remove the large variability in the microbial community across individuals, making it necessary to use larger number of animals for valid statistical analysis and interpretation. 
Therefore, cloned animals could have the potential of becoming good models, by reducing the number of animals needed for an experimental study and providing a less variable population, however, more optimization is needed to improve the quality of the cloned animals.

In regard to obesity related gut microbiota, we did not observe any association between weight-gain and change in bacterial diversity, although there was more bacterial richness in obese pigs. Taken together; these results point to specific changes in the bacterial community over time in both the cloned and non-cloned control pigs.

To get a better profile of the gut microbial community in relation to obesity, we compared the relative abundance of the phyla Bacteroidetes and Firmicutes in the pigs from baseline and throughout the diet intervention period until endpoint. In the case of Firmicutes, we observed an increase in relative abundance of this phylum from baseline to endpoint, in both cloned and non-cloned pigs and found a positive correlation with Firmicutes and weight-gain. This increase in the abundance of the phylum Firmicutes with increase in weight is in agreement with observations made in other studies [15]. One study [29], point to a connection between alterations in energy intake and changes in gut microbiota such as increase in abundance of Firmicutes. Jumpertz and colleagues [21] found that a 20\% increase in abundance of Firmicutes resulted in an increase in energy harvest corresponding to approximately 150 kilo calories. This suggests that the bloom in bacteria belonging to the phylum Firmicutes contributes to promotion of obesity and maintenance of the obese state.

The relative abundance of Bacteroidetes in the cloned pigs decreased continuously through the diet intervention period but then began steadily to increase until the animals were euthanized. The same was observed in the non-cloned control pig group and eventually the relative abundance of Bacteroidetes at endpoint was not different from baseline. This was unexpected, as previously it has been shown that obese subjects have less Bacteroidetes compared to their leaner counterparts $[10,16,30]$. Furthermore, one study on humans under a weight loss regiment showed [15] an increase in Bacteroidetes. One explanation to the observations made in our study could be that the bacteria belonging to phylum Bacteroidetes somehow adapt to the HF/high-caloric diet and their number at endpoint eventually reaches the values observed at baseline. Hildebrandt et al. [29] demonstrated a decrease in Bacteroidetes and an increase in Firmicutes in the gut microbiota of mice independent of obesity but in relation to HF diet in mice [29], while other studies point to the association of HF diet and the changes in abundance of Firmicutes in mice [4]. Together, these studies suggest that the changes in gut microbiota could be due to the HF/high caloric diet and not the state of obesity. Even though we found a positive relation between weight-gain and changes in the relative abundance of Firmicutes, we cannot exclude the possibility that the changes were also in relation to HF/high-caloric diet. Therefore, the gut microbiota could be a potential therapeutic target to fight obesity.

\section{Conclusion}

Here we conclude that cloned pigs do not appear to have smaller inter-individual variation as compared to the sibling non-cloned pigs with regard to their gut microbiota, and because it is both time consuming and costly, they are not more suitable than conventional pigs for gut-microbiota-obesity related studies.

Our findings agree with the hypothesis that the dietinduced obesity is related to changes in the relative abundance of Firmicutes and Bacteroidetes and especially an increase in proportion of the bacteria belonging to the phyla Firmicutes. We also point to HF/highcaloric diet as a contributing factor that changes the gut microbial community. To our knowledge this is the first study that has investigated the effects of diet-induced obesity on gut-microbiota in cloned pigs. More investigation is needed to optimize the cloning of experimental animals which could eventually offer a more controlled experimental model.

\section{Additional files}

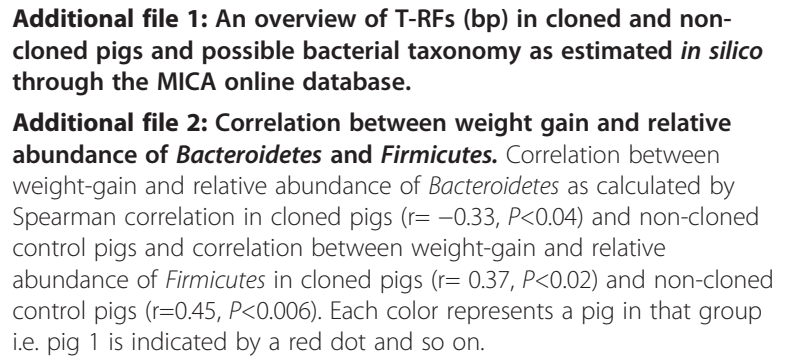

Additional file 2: Correlation between weight gain and relative abundance of Bacteroidetes and Firmicutes. Correlation between weight-gain and relative abundance of Bacteroidetes as calculated by Spearman correlation in cloned pigs $(r=-0.33, P<0.04)$ and non-cloned control pigs and correlation between weight-gain and relative abundance of Firmicutes in cloned pigs $(r=0.37, P<0.02)$ and non-cloned control pigs $(r=0.45, P<0.006)$. Each color represents a pig in that group i.e. pig 1 is indicated by a red dot and so on.

\section{Competing interest}

All authors declare no financial or any other competing interest.

\section{Authors' contributions}

$M B, L M$ and RP designed the study experiments. RP carried out the experimental work, data and statistical analysis and wrote the manuscript. A. D.A performed the statistical analysis on T-RFLP Shannon-Weaver diversity and PCA and contributed to writing of the manuscript. JS designed and conducted the animal and the diet-intervention experiments. All authors read, corrected and approved the final manuscript.

\section{Acknowledgements}

This work was supported by a grant from the Danish Strategic Research Council (FØSU 2101-06-0034), and The Danish Research Council FTP (096649307). We would like to thank Sophia Rasmussen and Joanna Amenuvor for excellent technical assistance.

\section{Author details}

${ }^{1}$ National Veterinary Institute, Technical University of Denmark, Bülowsvej 27 Frederiksberg C 1870, Denmark. ${ }^{2}$ Department of Food Science, University of Aarhus, Blichers Alle 20, Tjele 8830, Denmark. ${ }^{3}$ Present address: Chr. Hansen A/S, Bøge Alle 10-12, Hørsholm DK-2970, Denmark. 
Received: 27 August 2012 Accepted: 4 February 2013

Published: 7 February 2013

\section{References}

1. Stewart JA, Chadwick VS, Murray A: Investigations into the influence of host genetics on the predominant eubacteria in the faecal microflora of children. J Med Microbiol 2005, 54:1239-1242.

2. Zoetendal EG, Akkermans AD, WM K-V V, de Visser JA, de Vos WM: The host genotype affects the bacterial community in the human gastronintestinal tract. Microb Ecol Health Dis 2001, 13:129-134.

3. Turnbaugh PJ, Hamady M, Yatsunenko T, Cantarel BL, Duncan A, Ley RE, et al: A core gut microbiome in obese and lean twins. Nature 2009, 457:480-484

4. Murphy EF, Cotter PD, Healy S, Marques TM, O'Sullivan O, Fouhy F, et al: Composition and energy harvesting capacity of the gut microbiota: relationship to diet, obesity and time in mouse models. Gut 2010, 59:1635-1642.

5. Pang $X$, Hua $X$, Yang Q, Ding D, Che C, Cui L, et al: Inter-species transplantation of gut microbiota from human to pigs. ISME J 2007, 1:156-162.

6. Guilloteau P, Zabielski R, Hammon HM, Metges CC: Nutritional programming of gastrointestinal tract development. Is the pig a good model for man? Nutr Res Rev 2010, 23:4-22.

7. Neeb ZP, Edwards JM, Alloosh M, Long X, Mokelke EA, Sturek M: Metabolic syndrome and coronary artery disease in ossabaw compared with Yucatan swine. Comp Med 2010, 60(4):300-315. Ref Type: Abstract.

8. Sturek $\mathrm{M}: \mathrm{Ca} 2+$ Regulatory mechanisms of exercise protection against coronary artery disease in metabolic syndrome and diabetes. J Appl Physiol 2011, 111:573-586.

9. Clausen M, Christensen K, Hedemann M, Liu Y, Purup S, Schmidt M, et al: Metabolomic phenotyping of a cloned pig model. BMC Physiol 2011, 11:14.

10. Eckburg PB, Bik EM, Bernstein CN, Purdom E, Dethlefsen L, Sargent M, et al: Diversity of the human intestinal microbial flora. Science 2005, 308:1635-1638.

11. Ley RE, Hamady M, Lozupone C, Turnbaugh PJ, Ramey RR, Bircher JS, et al: Evolution of mammals and their gut microbes. Science 2008, 320:1647-1651.

12. Leser TD, Amenuvor JZ, Jensen TK, Lindecrona RH, Boye M, Moller K: Culture-independent analysis of gut bacteria: the pig gastrointestinal tract microbiota revisited. Appl Environ Microbiol 2002, 68:673-690.

13. Lamendella R, Santo Domingo J, Ghosh S, Martinson J, Oerther D: Comparative fecal metagenomics unveils unique functional capacity of the swine gut. BMC Microbiol 2011, 11:103.

14. Ley RE, Backhed F, Turnbaugh P, Lozupone CA, Knight RD, Gordon Jl: Obesity alters gut microbial ecology. Proc Natl Acad Sci 2005, 102:11070-11075.

15. Ley RE, Turnbaugh PJ, Klein S, Gordon J: Microbial ecology: human gut microbes associated with obesity. Nature 2006, 444:1022-1023.

16. Guo X, Xia X, Tang R, Zhou J, Zhao H, Wang K: Development of a real-time PCR method for firmicutes and bacteroidetes in faeces and its application to quantify intestinal population of obese and lean pigs. Lett Appl Microbiol 2008, 47:367-373.

17. Nadal I, Santacruz A, Marcos A, Warnberg J, Garagorri M, Moreno LA, et al: Shifts in clostridia, Bacteroides and immunoglobulin-coating fecal bacteria associated with weight loss in obese adolescents. Int J Obes 2008, 33:758-767.

18. Schwiertz A, Taras D, Schafer K, Beijer S, Bos NA, Donus C, et al: Microbiota and SCFA in lean and overweight healthy subjects. Obesity 2009, 18:190-195.

19. Fleissner CK, Huebel N, Abd El-Bary MM, Loh G, Klaus S, Blaut M: Absence of intestinal microbiota does not protect mice from diet-induced obesity. Br J Nutr 2010, 104:919-929.

20. Duncan SH, Lobley GE, Holtrop G, Ince J, Johnstone AM, Louis P, et al: Human colonic microbiota associated with diet, obesity and weight loss. Int J Obes 2008, 32:1720-1724.

21. Jumpertz R, Le DS, Turnbaugh PJ, Trinidad C, Bogardus C, Gordon Jl, et al: Energy-balance studies reveal associations between gut microbes, caloric load, and nutrient absorption in humans. Am J Clin Nutr 2011, 94:58-65.

22. Christensen KL, Hedemann MS, Jørgensen H, Stagsted J, Knudsen KE: Liquid chromatography-mass spectrometry based metabolomics study of cloned versus normal pigs Fed either restricted or Ad libitum highenergy diets. J Proteome Res 2012, 11:3573-3580.

23. Mølbak L, Johnsen $K$, Boye M, Jensen TK, Johansen M, Møller K, et al: The microbiota of pigs influenced by diet texture and severity of lawsonia intracellularis infection. Vet Microbiol 2008, 128:96-107.
24. Shyu C, Soule T, Bent S, Foster J, Forney L: MiCA: a Web-based tool for the analysis of microbial communities based on terminal-restriction fragment length polymorphisms of $16 \mathrm{~S}$ and $18 \mathrm{~S}$ rRNA genes. Microb Ecol 2007, 53:562-570.

25. Maidak BL, Cole JR, Lilburn TG, Parker CT Jr, Saxman PR, Farris RJ, et al: The RDP-II (ribosomal database project). Nucleic Acids Res 2001, 29:173-174.

26. Andersen AD, Mølbak L, Michaelsen KF, Lauritzen L: Molecular fingerprints of the human fecal microbiota from 9 to 18 months old and the effect of fish oil supplementation. J Pediatr Gastroenterol Nutr 2011, 53:303-309.

27. Bacchetti De Gregoris T, Aldred N, Clare AS, Burgess JG: Improvement of phylum- and class-specific primers for real-time PCR quantification of bacterial taxa. J Microbiol Methods 2011, 86:351-356.

28. Rødgaard T, Skovgaard KSJ, Heegaard PMH: Expression of innate immune response genes in liver and three types of adipose tissue in cloned pigs. Cell Reprograming 2012, 14:407-417.

29. Hildebrandt MA, Hoffmann C, Sherrill-Mix SA, Keilbaugh SA, Hamady M, Chen $Y Y$, et al: High-Fat diet determines the composition of the murine Gut microbiome independently of obesity. Gastroenterology 2009, 137:1716-1724.

30. Ley RE, Peterson DA, Gordon Jl: Ecological and evolutionary forces shaping microbial diversity in the human intestine. Cell 2006, 124:837-848.

doi:10.1186/1471-2180-13-30

Cite this article as: Pedersen et al:: Changes in the gut microbiota of cloned and non-cloned control pigs during development of obesity: gut microbiota during development of obesity in cloned pigs. BMC Microbiology 2013 13:30.

\section{Submit your next manuscript to BioMed Central and take full advantage of:}

- Convenient online submission

- Thorough peer review

- No space constraints or color figure charges

- Immediate publication on acceptance

- Inclusion in PubMed, CAS, Scopus and Google Scholar

- Research which is freely available for redistribution
C BioMed Central 